\title{
G

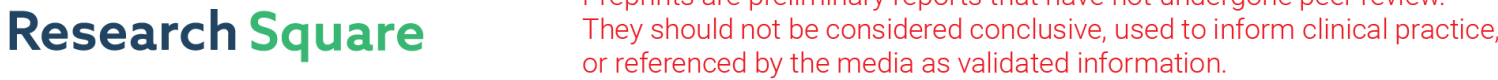 \\ Pseudogene OCT4-pg5 Upregulates OCT4B Expression To Promote Bladder Cancer Progression By Competing With miR-145
}

\section{Wuer Zhou}

General Hospital of Southern Theatre Command

\section{Yue Yang}

General Hospital of Southern Theatre Command https://orcid.org/0000-0002-2478-1601

Wei Wang ( $\square$ wangweiccc@hotmail.com )

General Hospital of Southern Theatre Command https://orcid.org/0000-0001-7237-1159

\section{Chenglin Yang}

General Hospital of Southern Theatre command

\section{Zhi Cao}

General Hospital of Southern Theatre command

Xiaoyu Lin

General Hospital of Southern Theatre Command

\section{Bangqi Wang}

General Hospital of Southern Theatre Command

\section{Yuansong Xiao}

General Hospital of Southern Theatre Command

Xiaoming Zhang

General Hospital of Southern Theatre Command

\section{Primary research}

Keywords: OCT4-pg5, OCT4B, miR-145, competing endogenous RNA, bladder cancer

Posted Date: September 9th, 2021

DOI: https://doi.org/10.21203/rs.3.rs-832388/v1

License: (c) (i) This work is licensed under a Creative Commons Attribution 4.0 International License. Read Full License 


\section{Abstract \\ Background}

Octamer-binding transcription factor 4 pseudogene 5 (OCT4-pg5) contributes to tumor progression in many cancer types, but contributions to bladder cancer (BC) have not been investigated.

\section{Methods}

Real-time quantity PCR (RT-qPCR) was performed to measure OCT4-pg5 and OCT4B expressions in different bladder cell lines and different grades of cancer. The effects of OCT4-pg5, OCT4B and miR-145 on proliferation and metastasis were determined by in vitro and in vivo experiments. Luciferase reporter assay was carried out to reveal the interaction among OCT4-pg5, OCT4B and miR-145. Flow cytometry was performed to explore the effects of OCT4-pg5 and OCT4B expression on the cell cycle stage distribution of T24 cells.

\section{Results}

OCT4-pg5 expression was significantly increased in BC cell lines, which was correlated with OCT4B expression and advanced tumor grade. Overexpression of OCT4-pg5 and OCT4B promoted the proliferation and invasion of BC cells, while miR-145 suppressed these activities. Mechanically, OCT4-pg5 $3^{\prime}$ untranslated region ( $3^{\prime} \mathrm{UTR}$ ) competed for miR-145, thereby increasing OCT4B expression. In addition, OCT4-pg5 promoted EMT by activating the Wnt/ $\beta$-catenin pathway and upregulating the expression levels of matrix metalloproteinases (MMPs) 2 and 9 as well as transcription factors zinc finger E-box binding homeobox (ZEB) 1 and 2. Furthermore, elevated expression of OCT4-pg5 and OCT4B reduced the sensitivity of $\mathrm{BC}$ cells to cisplatin by reducing apoptosis and increasing the proportion of cells in $\mathrm{G} 1$.

\section{Conclusions}

These findings indicate that OCT4-pg5/miR-145/OCT4B axis promotes the progression of BC by inducing EMT via Wnt/ $\beta$-catenin pathway and enhances the cisplatin resistance. It could be prospect for the therapeutic approaches for BC.

\section{Background}

Bladder cancer (BC) is one of the most common urological malignant neoplasms, with approximately 570,000 new cases diagnosed in 2020 [1]. Incidence and mortality of BC are positively correlated with smoking and GDP per capita [2], so incidence is expected to continue rising in developing countries. Moreover, $\mathrm{BC}$ is characterized by high rates of recurrence, metastasis, and insensitivity to chemotherapy 
[1]. Consequently, it is critical to elucidate the molecular pathogenesis of BC to identify more efficacious treatment targets.

Octamer-binding transcription factor 4 (OCT4), one of the POU domain-containing family of transcription factors, is implicated in the pathogenesis of multiple cancer types. The OCT4 gene can generate at least three distinct mRNA transcripts and proteins by alternative splicing and alternative translation initiation $[3,4]$. Hypoxia stimulates a short OCT4 isoform, OCT4B, via a hypoxia inducible factor (HIF)2a-dependent pathway to induce epithelial-mesenchymal transition (EMT) and facilitate cancer dissemination [5-7]. Further, OCT4B is highly expressed in bladder cancer (BC) and glioblastoma cells, and expression is correlated with poorer histopathological grade and clinical prognosis $[8,9]$, but its pathogenic function in $\mathrm{BC}$ is still unknown.

MicroRNA-145 (miR-145, chromosome 5q) functions as a tumor-suppressor in multiple cancers, including non-small-cell lung, bladder, and colorectal cancer, by downregulating its oncogenes [10]. In addition, miR145 can bind directly to the 3'untranslated region (3'UTR) of OCT 4 mRNA, thereby inhibiting the proliferation, infiltration, and migration of breast cancer [10], while it is still lack of reports in BC.

Long non-coding RNAs (IncRNAs), defined as untranslated RNA transcripts longer than 200 nucleotides, are now recognized as important regulators of tumorigenesis and progression [11], and are considered potential biomarkers for the early detection, diagnosis, and prognosis of BC [12]. Pseudogenes are nonfunctional copies of genes that also produce IncRNAs [13], and it is now recognized that pseudogene IncRNAs can regulate tumor progression, mainly by competing with miRNAs for binding to parent genes [14] and thereby interfering with miRNA-mediated gene suppression. For instance, OCT4-pg5 is an OCT4 pseudogene typically transcribed in cancer tissues that may upregulate OCT4 expression [15] by acting as an 'RNA sponge' to prevent inhibition by miR-145 [16]. However, the functions of OCT4-pg5, OCT4B, and miR-145 in BC have not been established.

In this study, we report that OCT4-pg5 expression is elevated in BC cells and tissues concomitantly with OCT4B, and that higher expression of both gene is strongly associated with more advanced pathological grade and stage. Subsequent functional studies including cell viability, cell migration, and Luciferasebased gene expression assays further indicated that OCT4-pg5 acts as a competing endogenous RNA (ceRNA) promoting OCT4B expression by sponging miR-145, thereby interfering with miR-145-mediated OCT $4 \mathrm{~B}$ downregulation and increasing $\mathrm{BC}$ cell metastatic capacity and drug resistance.

\section{Materials And Methods}

\section{Cell culture and clinical samples}

Five human bladder cancer cell lines (T24, EJ, BIU-87, 5637, and TCC-SUP) and an immortalized human bladder epithelial cell line (SV-HUC-1) were obtained from the American Type Culture Collection (ATCC, Manassas, VA, USA). The human bladder cancer cell line BIU-87 was preserved by our department (Department of Urology, General Hospital of Southern Theater Command, Guangzhou, China). Cells were 
maintained in RPMI 1640 (Gibco, Grand Island, NY) or DMEM (Gibco) supplemented with $10 \%$ fetal bovine serum (FBS; Gibco) in an atmosphere of $5 \% \mathrm{CO}_{2}$ at $37^{\circ} \mathrm{C}$.

A total of 140 human bladder cancer tissue samples and 34 adjacent bladder epithelial tissue samples were obtained from the General Hospital of Southern Theater Command (China) from February 2016 to October 2019. Inclusion criteria were confirmed non-muscle-invasive bladder cancer (NMIBC, $n=70$ ) or muscle-invasive bladder cancer (MIBC, $n=70$ ), while the exclusion criterion was metastasis before surgery. All patients provided informed written consent, and the study was approved by the Institute Research Ethics Committee, General Hospital of Southern Theater Command, China.

\section{Plasmid construction and transfection}

For OCT4-pg5 and OCT4B overexpression, the portion of the OCT4-pg5 3'UTR and OCT4B mRNA containing the miR-145 binding site were introduced into the pcDNA3.1(+) vector (Invitrogen, Carlsbad, CA). Target gene PCR products were digested with BamHI//Notl and cloned into the vectors, followed by DNA sequence verification. Human OCT4-pg5 siRNAs (si-OCT4-pg5), OCT4B siRNAs (si-OCT4B), miR-145 inhibitors, miR-145 mimics, and appropriate negative controls were acquired from Vipotion Biotechnology (Guangzhou, China). For in vitro assays, si-OCT4-pg5, si-OCT4B, miR-145 mimics, and control [empty pcDNA3.1(+)] were transfected into T24 cells, while pcDNA3.1(+)/OCT4-pg5, miR-145 mimics, pcDNA3.1(+)/OCT4-pg5 plus miR-145 mimics, pcDNA3.1(+)/OCT4B, or control vectors were transfected into 5637 cells as indicated using Lipofectamine 2000 reagent (Invitrogen) according to the manufacturer's protocol.

\section{RNA extraction and real-time PCR analysis}

Total RNA was extracted from tissues or cells using TRIZOL reagent (Invitrogen Life Technologies) according to the manufacturer's instructions. Real-time PCR was performed using a Stratagene Mx3000P Real-time PCR System (Applied Biosystems, Agilent Stratagene, America) and Bestar qPCR RT Kit (DBI Bioscience, Shanghai, China). The amplification procedure was as follows: $94{ }^{\circ} \mathrm{C}$ for $2 \mathrm{~min}$, followed by 40 cycles of $94{ }^{\circ} \mathrm{C}$ for $20 \mathrm{~s}, 58^{\circ} \mathrm{C}$ for $20 \mathrm{~s}$, and $72{ }^{\circ} \mathrm{C}$ for $20 \mathrm{~s}$. A dissociation step was performed to generate a melting curve for confirmation of amplification specificity. For miR-145, U6 was used as the internal reference gene, while b-actin was used as the reference for other genes. Relative gene expression levels were calculated using the $2^{-\Delta \Delta C t}$ method. All primers used were designed and produced by Vipotion Biotechnology, and are listed in Table S6.

\section{Luciferase reporter assay}

The wild-type 3'UTR sequence of OCT4 containing the putative miR-145 binding site was cloned into psiCHECK2 (Promega, Madison, WI, USA) to construct a 3'UTR luciferase reporter. For miRNA target analysis, cell lines (HEK293 and HepG2) were transfected with the luciferase reporter and co-transfected with empty vector (control), miR-145, wild-type OCT4-pg5 (wt-OCT4-pg5), wild-type OCT4-pg5 plus miR145 or empty (control), miR-145, mut-type OCT4-pg5 (mut-OCT4-pg5), or mutant-type OCT4-pg5 plus miR- 
145 plasmid as indicated. The luciferase activities were measured $48 \mathrm{~h}$ after transfection using a DualLuciferase Reporter Assay Kit (Promega) according to the manufacturer's instructions.

\section{Immunofluorescence staining}

After transfection, T24 or 5637 cells were grown on glass chamber slides to $90 \%$ confluence, fixed with $4 \%$ paraformaldehyde for 20 min, permeabilized with $0.1 \%$ Triton X-100 in phosphate-buffered saline (PBS) for $30 \mathrm{~min}$, and blocked with $3 \%$ bovine serum albumin (BSA) in PBS for $1 \mathrm{~h}$ at room temperature. Cells were then incubated with anti-OCT4B, anti- $\beta$-catenin, anti-E-cadherin, anti-vimentin, and (or) antiSnail antibodies overnight at $4{ }^{\circ} \mathrm{C}$. Immunolabeled cells were incubated with FITC-conjugated secondary antibody (Bioworld, Atlanta, GA, USA) for $1 \mathrm{~h}$ and finally counterstained with DAPI for $15 \mathrm{~min}$. Staining patterns were examined and captured using a laser scanning confocal microscope.

\section{Western blotting}

Total cellular protein was extracted in lysis buffer (Beyotime; Shanghai, China) and quantified using the Bradford method. Proteins were separated by $10 \%$ sodium dodecyl sulfate-polyacrylamide gel electrophoresis (SDS-PAGE) and transferred electrophoretically onto polyvinylidene difluoride membranes (Millipore, USA). The membranes were incubated overnight at $4{ }^{\circ} \mathrm{C}$ with the following primary antibodies: anti-OCT4B, anti-N-cadherin, anti- $\beta$-catenin (all from Santa Cruz Biotechnology; Dallas, TX, USA), antivimentin, anti-Snail, anti-E-cadherin, anti-MMP2, anti-MMP9, anti-ZEB1, anti-ZEB2, and anti-GAPDH (all from Affbiotech; Shanghai, China). Protein levels were quantified by densitometry using Image-Pro Plus 6.0 .

\section{Cell viability assay}

Cell proliferation was measured using the Cell Counting Kit-8 (Dojindo, Cat. No.CK04) according to the manufacturer's instructions. T24 or 5637 cells were seeded in 96 -well culture plates at $1 \times 10^{4}$ cells/well, cultured overnight, transfected with the indicated plasmids for $48 \mathrm{~h}$, washed, and cultivated in complete medium for the indicated growth period $(0,24,48$, and $72 \mathrm{~h}$ ). Cells were then treated with $10 \mathrm{ml} /$ well Cell Counting Kit-8 solution for $4 \mathrm{~h}$, and total viable cell number estimated by the absorbance at $450 \mathrm{~nm}$ using a microplate reader (Thermo Fisher Scientific, Multiskan MK3).

\section{Migration and invasion assays}

In vitro migration and invasion assays were conducted using uncoated and Matrigel-coated 24-well transwell chambers (pore size of $8 \mu \mathrm{M}$; Costar, Corning, NY, USA), respectively, according to the manufacturer's instructions. T24 or 5637 cells were plated at $5 \times 10^{5} /$ well in the upper chambers of 24 -well transwell plates with FBS-free medium, while the bottom chambers were filled with culture medium containing $20 \% \mathrm{FBS}$. After $48 \mathrm{~h}$ of incubation at $37^{\circ} \mathrm{C}$ in a humidified $5 \% \mathrm{CO}_{2}$ atmosphere, cells in the upper chambers were removed, and migratory or invasive cells were stained with $0.1 \%$ crystal violet 
solution for $15 \mathrm{~min}$. Total cell numbers from 6 randomly chosen fields per membrane were quantified at 200x magnification. Mean cell numbers from triplicate assays were calculated for each condition.

\section{Wound-healing assay}

After transfection, T24 cells were seeded into 6 -well plates at $2 \times 10^{5}$ cells/well and allowed to grow to $90 \%$ confluence in complete medium. Cell monolayers were then scratched (wounded) using a sterile plastic pipette tip $(200 \mathrm{ml})$, washed three times with PBS to remove cell debris, and incubated in serumfree medium for $24 \mathrm{~h}$. Cells migrating into the wound area were photographed under an inverted microscopy at designated times, and the average distance of migration was calculated.

\section{Colony formation assays}

Transfected T24 or 5637 cells were seeded in 6-well plates at 500 cells/well and cultured for 10 days. Colonies were fixed in paraformaldehyde, stained with crystal violet, photographed, and counted.

\section{Cell cycle and apoptosis analyses}

Cell cycle and apoptosis analyses were conducted by flow cytometry (FCM) using a FACS Calibur flow cytometer (BD Biosciences, San Jose, CA, USA). For cell cycle analysis, transfected cells were plated at $5 \times 105 / \mathrm{mL}$ in 6-well plates, incubated for $4-6 \mathrm{~h}$ in complete medium, washed with PBS, and then incubated in fresh complete medium for another $48 \mathrm{~h}$. Cells were harvested, centrifuged, and fixed in $70 \%$ cold ethanol for $2 \mathrm{~h}$. DNA staining was conducted using $300 \mathrm{ml} /$ well cell cycle staining kit solution (Vazyme Biotech, Nanjing, China) for at least 15 min under darkness. For cell apoptosis analysis, cells transfected as indicated were stained using an AnnexinV-FITC Apoptosis Detection Kit (Vazyme Biotech). The proportion of apoptotic cells was analyzed by FCM using Cell Quest software.

\section{Xenograft tumor model}

Six-week-old BALB/c nude mice were acquired from the Model Animal Research Center of Southern Medical University. All animal procedures were performed according to protocols approved by the Institutional Animal Care and Use Committee of the General Hospital of Southern Theater Command. To establish the xenograft tumor model, $5 \times 10^{6}$ T24 cells stably transfected with si-OCT4-pg5 or NC-si-RNA, and $5 \times 10^{6} 5637$ cells stably transfected with pcDNA3.1(+)/OCT4-pg5 or NC-pcDNA3.1(+) were injected subcutaneously in the left flank of separate BALB/c nude mouse groups (5 mice per group). Tumor volumes were evaluated every three days and calculated according to the equation

$V\left(m m^{3}\right)=\frac{A \times B^{2}}{2}$,

where $A$ is the largest diameter and $B$ is the perpendicular diameter. After 27 days, mice were sacrificed and tumors were isolated and weighted. 


\section{Statistical analysis}

All statistical analyses were performed using SPSS 22.0, and graphs were constructed using GraphPad Prism 5. Results are expressed as mean \pm SD of at least three independent experiments. Group means were compared using independent samples $t$-test. Categorial data were analyzed by the chi-square test or Fisher exact test. $P<0.05$ (two-tailed) was regarded as statistically significant for all tests.

\section{Results}

\section{Elevated OCT4-pg5 expression in BC and positive correlations with OCT4B expression and pathological grade}

To identify potential contributions of OCT4-pg5 to BC development and pathological grade, we first compared mRNA expression levels between BC tissues and normal adjacent tissues, as well as between five BC cell lines (T24, EJ, 5637, BIU-87, and TCCSUP) and a normal bladder cell line (SV-HUC-1). Expression of OCT4-pg5 was significantly higher in cancer tissues than adjacent normal tissue as measured by RT-PCR (Fig. 1a), and higher expression level was associated with advanced pathological grade (Fig. 1b), suggesting OCT4-pg5 as a potential prognostic marker. Among BC cell lines, OCT4-pg5 expression was highest in T24 cells (Fig. 1C). Moreover, OCT4-pg5 expression was strongly and positively correlated with OCT4B expression in BC tissues (Fig. 1d), suggesting possible co-regulation.

We further investigated if miR-145 and OCT4 isoform expression levels were associated with pathological or clinical features. Indeed, low miR-145 and high OCT4 expression levels were significantly correlated with greater tumor clinical stage and pathological grade, but not with patient sex, age, or smoking history (Additional files 1-5: Table S1-5).

\section{MicroRNA(miR)-145 repressed migration and invasion of T24 cells in vitro}

To evaluate the biological significance of miR-145 in BC, we transfected T24 cells with vectors expressing miR-145 inhibitors or miR-145 mimics, and tested for changes in migration and invasive capacity. Woundhealing assays showed that miR-145 overexpression significantly reduced T24 cell migration (Fig. 2a), while transwell assays showed that miR-145 downregulation significantly enhanced the number of cells migrating and invading from the top transwell chamber into untreated and Matrigel-coated membranes (Fig. 2b-c).

\section{OCT4-pg5 functioned as an oncogene in $\mathrm{BC}$ in vitro and in vivo}

To evaluate the effects of OCT4-pg5 on the oncogenic properties of BC cells, we tested for changes in the proliferation and invasive capacities of faster-growing T24 cells and slower-growing 5637 cells both in vitro and following inoculation in vivo. Transfection of T24 cells with si-OCT4-pg5 (knockdown group) significantly reduced both the proliferation rate and the number of cell colonies formed after $72 \mathrm{~h}$ compared to cultures transfected with control vector (Fig. 3a-c). Conversely, OCT4-pg5 transfection actually increased the proliferation and colony formation rates of 5637 cells (Fig. 3b-d). In addition, 
OCT4-pg5 knockdown significantly weakened the invasive capacity of T24 cells in transwell assays, while the invasive capacity of 5637 cells was enhanced (Fig. 3e-f). Mice injected with OCT4-pg5 overexpressing cells developed significantly larger tumors than mice injected with untransfected cells (Fig. 3g-i). Overexpression of miR-145 partially inhibited the enhanced proliferation, colony formation and invasion of 5637 cells induced by OCT4-pg5 overexpression (Fig. 3b, d, f). On the contrary, OCT4-pg5 overexpression partly reversed the suppression caused by miR-145 (Fig. 3b, d, f). These results suggest that OCT4-pg5 functions by altering the activity of miR-145.

\section{OCT4B promoted the proliferation, colony formation and invasion capacities of BC cells in vitro}

These same assays were conducted to assess the oncogenic functions of OCT4B. Knockdown of OCT4B significantly inhibited proliferation, while overexpression increased $\mathrm{BC}$ cell invasiveness in transwell assays (Fig. 3a, C, e), suggesting that OCT4B may promote cancer progression.

\section{The OCT4-pg5 3'UTR upregulated OCT4B expression by sequestering miR-145}

Sequence alignment revealed that the OCT4-pg5 3'UTR and OCT4B 3'UTR share a similar miR-145 binding site (Fig. 4a). Further, prediction of the secondary structures after miR-145 binding using RNAhybrid (https://bibiserv.cebitec.uni-bielefeld.de/ RNAhybrid) yielded identical free energy changes (-28.9 kcal/mol) for the OCT4-pg5 3'UTR and OCT4B 3'UTR (Fig. 4b). Moreover, luciferase assays showed that overexpression of wt-OCT4-pg5 in HEK293 and HepG2 cells significantly increased the activity of wild-type OCT4 3'UTR reporter, while inhibition of OCT4B expression by miR-145 was reversed upon transfection with OCT4-pg5 (Fig. 4c). However, these effects were not found following mut-OCT4-pg5 transfection (Fig. 4d). Collectively, these results suggest that OCT4-pg5 may compete with miR-145 for OCT 4B 3'UTR binding in BC, thereby allowing OCT4B upregulation.

To verify these results, we conducted qRT-PCR and western blot of lysates from transfected T24 and 5637 $B C$ cells and found that overexpression of miR-145 significantly downregulated both OCT4-pg 5 and OCT4B (Fig. 4e, f, i, j, k, I). Further, suppression of OCT4-pg5 and OCT4B expression in 5637 cells by miR145 transfection could be partly abolished by transfection of OCT4-pg5 overexpression plasmid (Fig. 4f, j, I). In addition, OCT4-pg5 knockdown significantly decreased OCT4B mRNA and protein expression in T24 cells (Fig. 4e, k). Immunofluorescence assays also revealed that OCT4B was located in the cytoplasm, and either OCT4-pg5 knockdown or miR-145 overexpression suppressed OCT4B expression (Fig. 4m), while OCT4-pg5 overexpression enhanced OCT4B expression (Fig. 4n).

\section{OCT4-pg5 promoted metastasis by regulating Epithelial-Mesenchymal Transition}

Epithelial-mesenchymal transition (EMT) promotes cancer progression by enhancing invasive capacity. To examine if OCT4-pg5 regulates EMT in BC, we measured the expression levels of EMT-associated transcription factors in T24 and 5637 cells overexpressing OCT4-pg5. Overexpression in T24 cells resulted in increased expression levels of E-cadherin mRNA (Fig. 5s) and protein (Fig. 5f), and lower expression levels of N-cadherin, vimentin, and Snail mRNAs (Fig. 5b-d) and proteins (Fig. 5g). In contrast, 
the pcDNA3.1(+)/OCT4-pg5 or pcDNA3.1(+)/OCT4B transfected 5637 cells expressed lower mRNA and protein levels of E-cadherin (Fig. $5 \mathrm{a}, \mathrm{f}$ ) and higher expression levels of $\mathrm{N}$-cadherin, vimentin, and Snail mRNAs (Fig. 5b-d) and proteins (Fig. 5g) compared to negative controls. It is noteworthy that OCT4B overexpression had an even stronger moderating effect on EMT markers than OCT4-pg5 overexpression. Immunofluorescence staining also revealed markedly increased expression of E-cadherin and decreased expression levels of vimentin and Snail following OCT4-pg5 knockdown (Fig. 5h-k).

\section{OCT4-pg5 and OCT4B regulated EMT via a Wnt/ $\beta$-catenin signaling pathway}

The Wnt/ $\beta$-catenin signaling pathway is implicated in the regulation of EMT, which in turn promotes metastasis [17]. To examine potential contributions of Wnt/ $\beta$-catenin signaling to EMT induction by OCT4-pg5 and OCT4B, we performed qRT-PCR, Western blotting, and immunofluorescence assays to measure changes in EMT signaling molecules in transfected T24 and 5637 cells. Downregulation of OCT4-pg5 and OCT4B reduced $\beta$-catenin expression, while OCT4-pg5 and OCT4B overexpression enhanced $\beta$-catenin expression (Fig. 5e, f, k). Moreover, expression levels of MMP2, MMP9, ZEB1, and ZEB2 were downregulated by OCT4-pg5 or OCT4B knockdown (Fig. $5 \mathrm{~g}$ ).

\section{OCT4-pg5 and OCT4B enhanced the resistance of T24 cells to cisplatin}

Cisplatin is one of the first-line treatments for BC [18]. We tested whether OCT4-pg5 or OCT4B could regulate the sensitivity of $B C$ cells to cisplatin. Treated with $85 \mathrm{mmol} / \mathrm{L}$ cisplatin for $48 \mathrm{~h}$, transfection with si-OCT4-pg5 or si-OCT4B significantly downregulated OCT4-pg5 and OCT4B mRNA expression levels as measured by qRT-PCR (Fig. 6a, b), and the expression levels of both OCT4B mRNA and protein were reduced by OCT4-pg5 knockdown (Fig. 6b, c).

Flow cytometry showed that OCT4-pg5 or OCT4B downregulation increased cisplatin-induced apoptosis of T24 cells (Fig. 6d). Similarly, OCT4-pg5 downregulation increased the proportion of cisplatin-treated T24 cells in G1 phase and decreased the proportion in S phase (Fig. 6e). In addition, si-OCT4-pg5 and siOCT 4B co-transfection had a stronger effect on cisplatin-induced cell apoptosis and cell cycle regulation than si-OCT4-pg5 or si-OCT4B transfection separately (Fig. 6e).

\section{Discussion}

The competitive regulatory interactions among ncRNAs collectively constitute ceRNA networks that if dysfunctional, may disrupt the complex molecular circuitry maintaining appropriate levels of cell proliferation and phenotype stability, culminating in tumorigenesis and progression [19]. Our study revealed that miR-145 can reduce the migratory and invasive capacities of T24 BC cells in vitro, consistent with previous studies on other cancer cell types [10]. Additionally, we found that the expression levels of OCT4-pg5 and OCT4B were upregulated in BC tissue samples and cell lines, and that OCT4-pg5 expression correlated with clinical and histopathological indices of prognosis, suggesting that OCT4-pg5 and OCT4B are oncogenic in BC as in other cancer types $[8,16,20,21]$. 
Pseudogenes are implicated in the initiation and progression of cancers, at least in part by acting as microRNA decoys that disrupt normal miRNA-mediated regulation of oncogene [22]. miR-145 can bind to OCT4 mRNA as well as to the OCT4 pseudogenes OCT4-pg1, 3, 4 and 5 [3], and recent reports have demonstrated that OCT4-pg4 can regulate OCT4 expression and compete with miR-145 in hepatocellular carcinoma [23]. Additionally, OCT4-pg5 acts as a miR-145 sponge in endometrial cancer, resulting in elevated OCT4 expression [16]. Similarly, PTENP1 has been shown to function as a ceRNA that competes for miRNAs targeting the PTENP1 3'UTR [24], while miR-145 can regulate OCT4 expression by targeting the OCT4 3'UTR in various cancer types $[25,26]$. Given that the OCT4-pg5 3'UTR and OCT4B 3'UTR share the same miR-145 binding site and show the same free energy change upon binding, it appears that OCT4-pg5 competes equally with miR-145 for the OCT4B 3'UTR but does not trigger mRNA degradation. Indeed, inhibition of OCT4B expression by miR-145 could be reversed by OCT4-pg5 overexpression. Collectively, these results strongly suggest that OCT4-pg5 acts as an oncogene by functioning as a ceRNA, thereby elevating parental OCT4B expression.

In several cancer types, overexpression of OCT4B was reported to prevent apoptosis [7] and increase migration, invasion, and extracellular matrix degradation capacities by inducing various EMT-related genes [27]. Further, several studies found that OCT4 upregulated the transcription factors ZEB1, ZEB2, and Snail by activating $\beta$-catenin, while miR-145 inhibited EMT by blocking the expression of OCT4, thereby downregulating the expression of Snail, ZEB1, and ZEB2 [28-30]. We also found that expressions of ZEB1, ZEB2, Snail, and two additional metastasis-related genes, MMP2 and MMP9, were upregulated in BC cells by OCT4B overexpression as well as by OCT4-pg5 overexpression. However, OCT4B demonstrated stronger EMT induction potential than OCT4-pg5, suggesting that OCT4-pg5 may induce EMT indirectly by regulating OCT4B expression. The Wnt/ $\beta$-catenin signaling pathway is known to promote metastasis by inducing EMT [17], and the LEF1/ $\beta$-catenin-dependent WNT signaling pathway can be activated by OCT4 [31]. Indeed, silencing $\beta$-catenin blocked Oct4/Nanog-mediated EMT [32]. In the current study, $\beta$-catenin was activated by OCT4-pg 5 and OCT4B, suggesting that OCT4-pg 5 and OCT4B may induce EMT via Wnt/ $\beta$-catenin signaling.

It was reported that OCT4 knockdown protected NSCLC cells from apoptosis and enhanced sensitivity to cisplatin treatment [33], while overexpression of OCT4 pro-moted the differentiation of lung cancer cells into SLCCs and increased cisplatin resistance [34]. Similarly in the current study, OCT4-pg5 and OCT4B expression protected T24 BC cells from cisplatin damage, while OCT4-pg5 knockdown increased cisplatin-induced apoptosis and reduced the proportion of G1 cells, possibly by disinhibiting OCT4 expression. Further studies are needed to identify the biological mechanism linking MMP2 and MMP9 upregulation to OCT4-pg5/miR-145/OCT4B axis activity and to confirm that this axis can regulate cisplatin sensitivity in $\mathrm{BC}$ cases, thereby altering disease progression and clinical outcome.

\section{Conclusions}

Our study demonstrates that OCT4-pg5 can promote oncogenesis by competing with miR-145 for binding to OCT4B mRNA, thereby disrupting miR-145-mediated OCT4B downregulation and leading to OCT4B 
overexpression, and ultimately greater BC cell proliferative and invasive capacity. These findings identify new potential therapeutic targets for BC.

\section{Abbreviations}

OCT4, Octamer-binding transcription factor 4;

OCT4-pg5, Octamer-binding transcription factor 4 pseudogene 5;

$\mathrm{BC}$, bladder cancer;

OCT4B, Octamer-binding transcription factor 4B;

MiR-145, MiRNA-145;

EMT, Epithelial-to-mesenchymal transition;

3'UTR, 3' untranslated regions;

CeRNAs, Competing endogenous RNAs;

LncRNAs, Long non-coding RNAs;

ATCC, American Type Culture Collection;

NMIBC, Non-muscle-invasive bladder tumors;

MIBC, Muscle-invasive bladder tumors;

PBS, Phosphate-buffered saline;

SDS-PAGE, Sodium dodecyl sulfate-polyacrylamide gel;

FCM, Flow cytometry.

\section{Declarations}

\section{Acknowledgements}

Not applicable.

\section{Authors' contributions}

WZE, YY and CLY prepared and wrote the original draft. WZE conducted the experiments and analyzed data. WW conceived the study. ZC, XYL, BQW, YSX, and XMZ validated the paper. WZE, YY and CLY 
contributed equally to this paper and should be considered as co-first author. All the authors have read and agreed to the final version of the manuscript.

\section{Funding}

This study was supported by the Guangzhou Science and Technology Plan Project Basic and Applied Basic Research Project (202002030030), the Guangdong Province Basic and Applied Basic Research Fund Project (2020A1515010044), and the National Science Fund subsidized project $\llbracket 81372744 \rrbracket$.

\section{Availability of data and materials}

The data presented in this study are available on request from the corresponding author.

\section{Ethics approval and consent to participate}

All the patients were informed of consent forms, and the study was approved by the Institute Research Ethics Committee, General Hospital of Southern Theater Command, China. All animal procedures were performed according to the protocols approved by the Institutional Animal Care and Use Committee at General Hospital of Southern Theater Command.

\section{Consent for publication}

All human tissue samples were obtained with informed consent from all subjects.

\section{Competing interests}

The authors declare no conflict of interest.

\section{References}

1. Sung H, Ferlay J, Siegel RL, Laversanne M, et al. Global Cancer Statistics 2020: GLOBOCAN Estimates of Incidence and Mortality Worldwide for 36 Cancers In 185 Countries. CA Cancer J Clin. 2021;0:1-41.

2. Teoh JY, Huang J, Ko WY, et al. Global trends of bladder cancer incidence and mortality, and their associations with tobacco use and gross domestic product per capita. Eur Urol. 2020;78:893-906.

3. Patra SK. Roles of OCT4 in pathways of embryonic development and cancer progression. Mechanisms of Ageing Development. 2020;189:111286.

4. Wang X, Zhao Y, Xiao Z, et al. Alternative translation of OCT4 by an internal ribosome entry site and its novel function in stress response. Stem Cells. 2009;27:1265-75.

5. Poursani EM, Mehravar M, Shahryari A, Mowla SJ, Mohammad Soltani B. Alternative splicing generates different 5' UTRs in OCT4B variants. Avicenna J Med Biotechnol. 2017;9:201-4.

6. Lin SC, Chung $\mathrm{CH}$, Chung $\mathrm{CH}$, et al. OCT4B mediates hypoxia-induced cancer dissemination. Oncogene. 2019;38:1093-105. 
7. Meng L, Hu H, Zhi H, et al. OCT4B regulates p53 and p16 pathway genes to prevent apoptosis of breast cancer cells. Oncol Lett. 2018;16:522-8.

8. Poursani EM, Mehravar M, Mohammad Soltani B, Mowla SJ, Trosko JE. A Novel variant of OCT4 entitled OCT4B3 is expressed in human bladder cancer and astrocytoma cell lines. Avicenna J Med Biotechnol. 2017;9:142-5.

9. Choi SH, Kim JK, Jeon HY, Eun K, Kim H. OCT4B Isoform promotes anchorage-independent growth of glioblastoma cells. Mol Cells. 2019;42:135-42.

10. Xu WX, Liu Z, Deng F, et al. MiR-145: a potential biomarker of cancer migration and invasion. Am J Transl Res. 2019;11:6739-53.

11. Chi Y, Wang D, Wang J, Yu W, Yang J. Long non-coding RNA in the pathogenesis of cancers. Cells. 2019;8:1015.

12. Taheri M, Omrani MD, Ghafouri-Fardsun S. Long non-coding RNA expression in bladder cancer. Biophys Rev. 2018;10:1205-13.

13. Hirotsune $S$, Yoshida $N$, Chen $A$, et al. An expressed pseudogene regulates the messenger-RNA stability of its homologous coding gene. Nature. 2003;423:91-6.

14. Hu X, Yang L, Mo Y. Role of pseudogenes in tumorigenesis. Cancers (Basel). 2018;10:256.

15. Poursani EM, Mohammad SB, Mowla SJ. Differential expression of OCT4 pseudogenes in pluripotent and tumor cell lines. Cell J. 2016;18:28-36.

16. Bai M, Yuan M, Liao H, et al. OCT4 pseudogene 5 upregulates OCT4 expression to promote proliferation by competing with miR-145 in endometrial carcinoma. Oncol Rep. 2015;33:1745-52.

17. Khan AQ, Ahmed El, Elareer NR, Junejo K, Steinhoff M, Uddin S. Role of miRNA-regulated cancer stem cells in the pathogenesis of human malignancies. Cells. 2019;8:840.

18. Yoshida T, Kates M, Fujita K, Bivalacqua TJ, McConkey DJ. Predictive biomarkers for drug response in bladder cancer. Int J Urol. 2019;26:1044-53.

19. Chan JJ, Tay Y. Noncoding RNA. RNA Regulatory networks in cancer. Int J Mol Sci. 2018;19:1310.

20. Choi SH, Kim JK, Jeon HY, Eun K, Kim H. OCT4B Isoform promotes anchorage-independent growth of glioblastoma cells. Mol Cells. 2019;42:135-42.

21. Soheili S, Asadi MH, Farsinejad A. Distinctive expression pattern of OCT4 variants in different types of breast cancer. Cancer Biomark. 2017;18:69-76.

22. Lou W, Ding B, Fu P. Pseudogene-derived IncRNAs and their miRNA sponging mechanism in human cancer. Front Cell Dev Biol. 2020;8:85.

23. Wang L, Guo ZY, Zhang R, et al. Pseudogene OCT4-pg4 functions as a natural microRNA sponge to regulate OCT4 expression by competing for miR-145 in hepatocellular carcinoma. Carcinogenesis. 2013;34:1773-81.

24. Poliseno L, Salmena L, Zhang J, Carver B, Haveman WJ, Pandolfi PP. A coding-independent function of gene and pseudogene mRNAs regulates tumour biology. Nature. 2010;465:1033-8. 
25. Matsushita R, Yoshino H, Enokida H, Goto Y, Miyamoto K, Yonemori M, et al. Regulation of UHRF1 by dual-strand tumor-suppressor microRNA-145 (miR-145-5p and miR-145-3p): inhibition of bladder cancer cell aggressiveness. Oncotarget. 2016;7:28460-87.

26. Wu Y, Liu S, Xin H, et al. Up-regulation of microRNA-145 promotes differentiation by repressing OCT4 in human endometrial adenocarcinoma cells. Cancer. 2011;117:3989-98.

27. Zhou JM, Hu SQ, Jiang H, et al. OCT4B1 promoted EMT and regulated the self-renewal of CSCs in CRC: effects associated with the balance of miR-8064/PLK1. Mol Ther Oncolytics. 2019;15:7-20.

28. Zhao H, Kang X, Xia X, et al. MiR-145 suppresses breast cancer cell migration by targeting FSCN-1 and inhibiting epithelial-mesenchymal transition. Am J Transl Res. 2016;8:106-3114.

29. Gao Y, Zhang Z, Li K, et al. Linc-DYNC2H1-4 promotes EMT and CSC phenotypes by acting as a sponge of miR-145 in pancreatic cancer cells. Cell Death Dis. 2017;8:e2924.

30. Li C, Lu L, Feng B, et al. The lincRNA-ROR/miR-145 axis promotes invasion and metastasis in hepatocellular carcinoma via induction of epithelial-mesenchymal transition by targeting ZEB2. Sci Rep. 2017;7:4637.

31. Sun L, Liu T, Zhang S, Guo K, Liu Y. Oct4 induces EMT through LEF1/ $\beta$-catenin dependent WNT signaling pathway in hepatocellular carcinoma. Oncol Lett. 2017;13:2599-606.

32. Liu $L$, Zhu $H$, Liao $Y$, et al. Inhibition of Wnt/ $\beta$-catenin pathway reverses multi-drug resistance and EMT in Oct4+/Nanog + NSCLC cells. Biomed Pharmacother. 2020;127:110225.

33. Liu X, Ma M, Duan X, Zhang H, Yang M. Knockdown of OCT4 may sensitize NSCLC cells to cisplatin. Clin Transl Oncol. 2017;19:587-92.

34. Chiou SH, Wang ML, Chou YT, et al. Co-expression of Oct4 and Nanog enhances malignancy in lung adenocarcinoma by inducing cancer stem cell-like properties and epithelial-mesenchymal transdifferentiation. Cancer Res. 2010;70:10433-44.

\section{Figures}


a
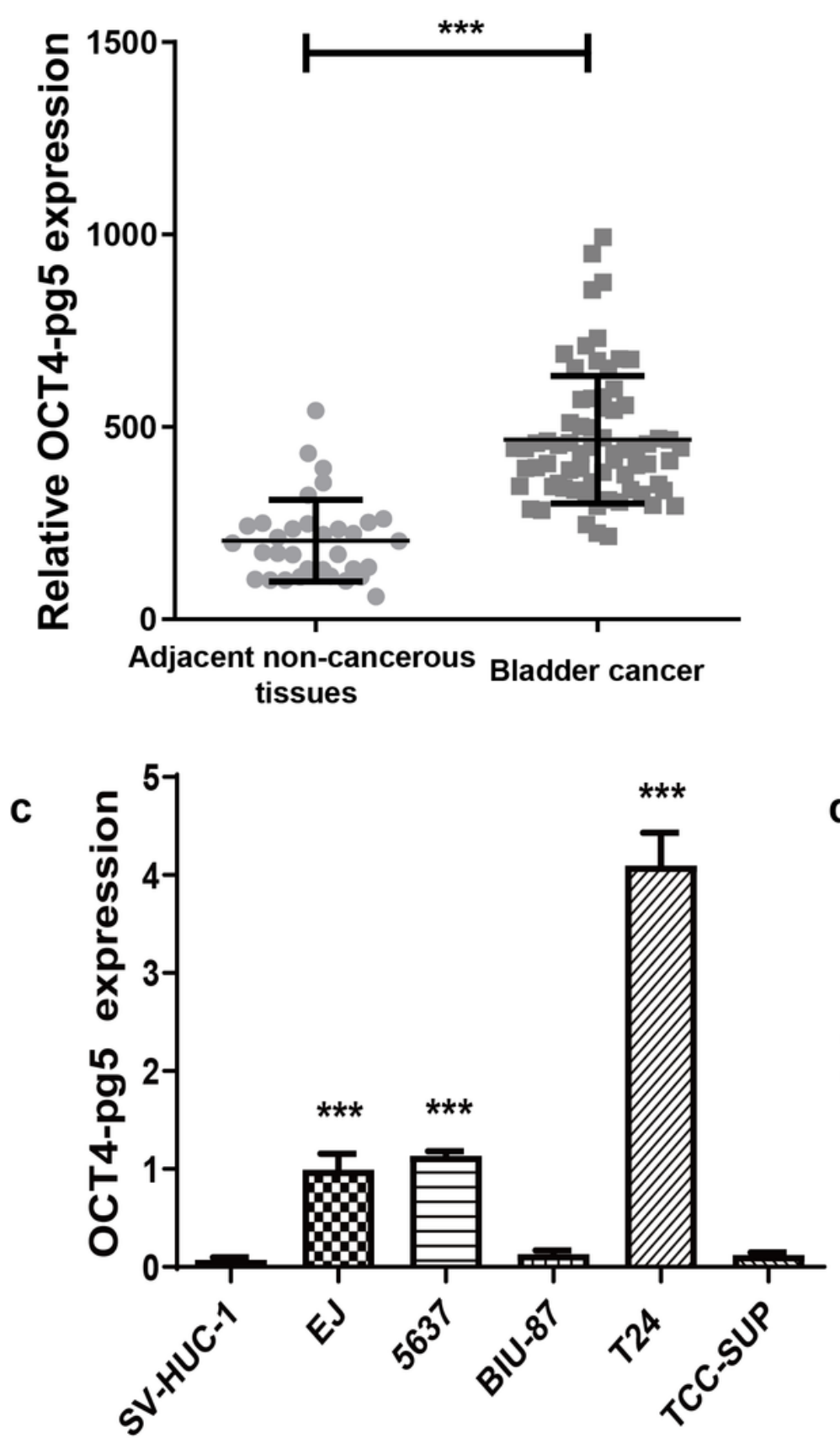

b
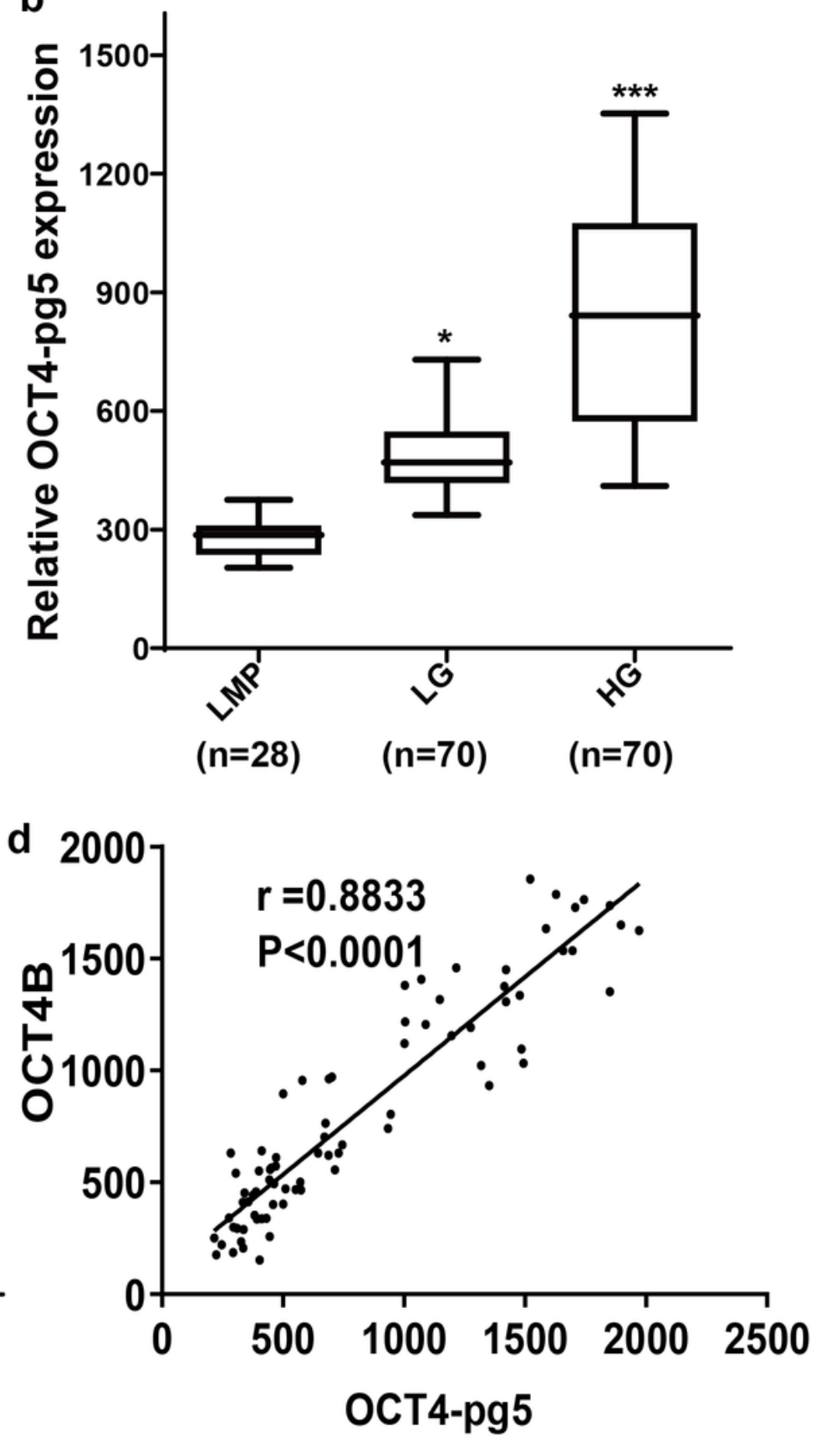

Figure 1

OCT4-pg5 expression is upregulated in human bladder cancer (BC) cells and tissues, co-regulated with OCT4B mRNA expression and highest in advanced cases. A, OCT4-pg5 expression in BC tissues $(n=140)$ and adjacent non-cancerous tissues(n=34). B, OCT4-pg5 expression level in different histological grades of BC (Papillary urothelial neoplasms of low malignant potential, PUN-LMP; low grade, LG; high grade, HG). C, OCT4-pg5 expression in five BC cell lines (T24, EJ, 5637, BIU-87 and TCCSUP) and a normal bladder cell line (SV-HUC-1). D, Relationship between OCT4-pg5 and OCT4B mRNA expression levels in BC tissues. All expression levels were measured by RT-PCR. Data were compared using independent samples t-test and Pearson's correlation test. 
a

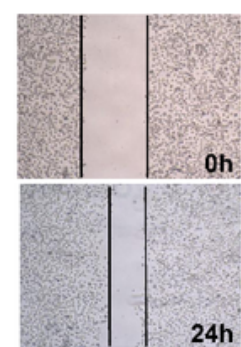

NC mimics

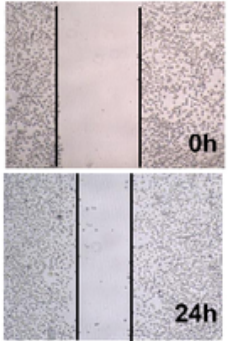

miR-145 mimics

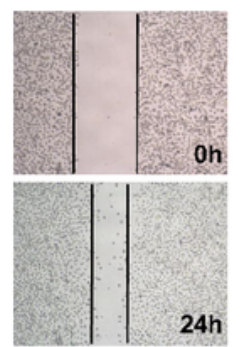

NC inhibitor

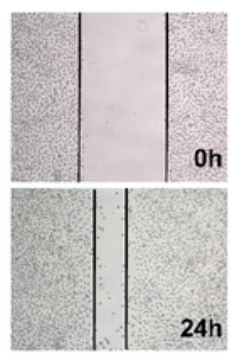

miR-145 inhibitor

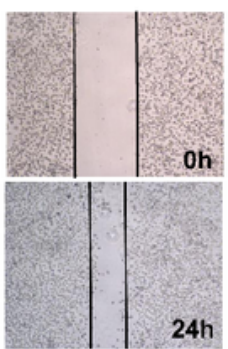

Control

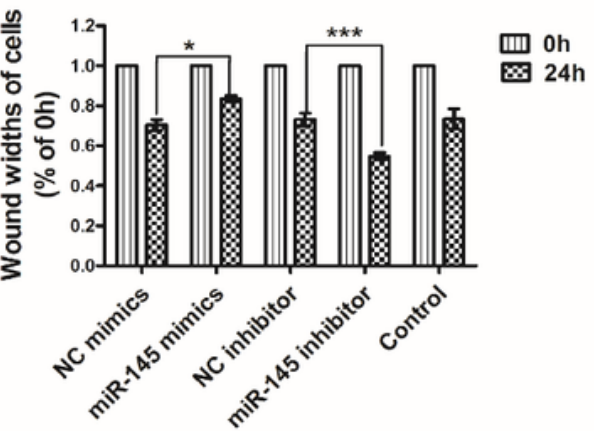

b

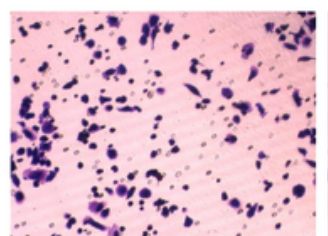

NC mimics

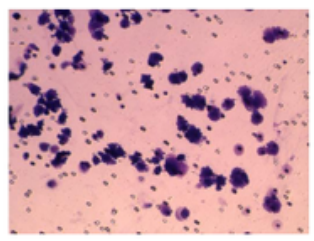

miR-145 mimics

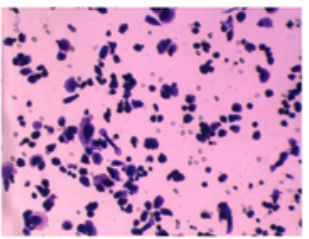

NC inhibitor

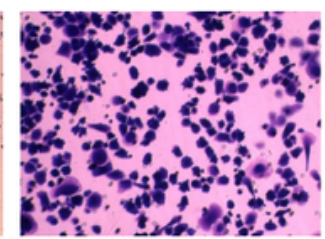

miR-145 inhibitor

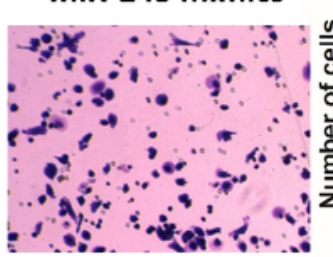

Control
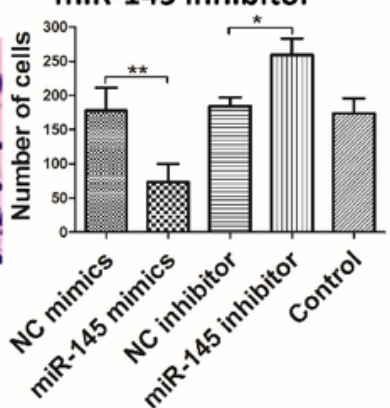
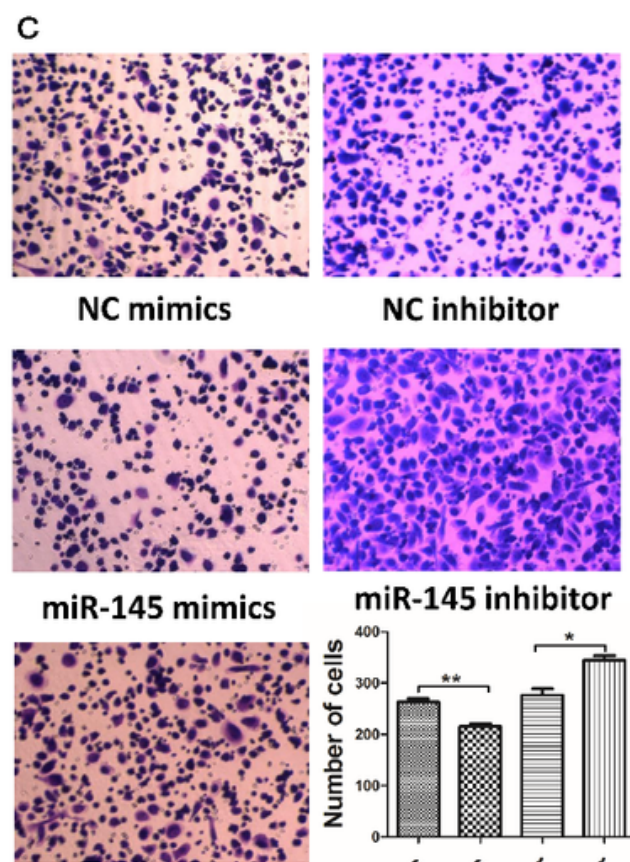

Control

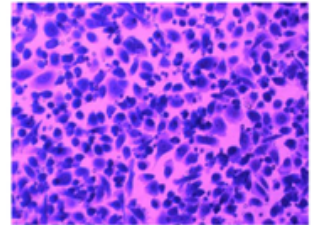

miR-145 inhibitor
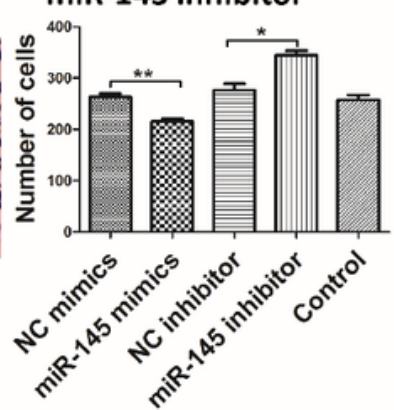

\section{Figure 2}

Overexpression of miR-145 reduces the migration and invasion potential of T24 BC cells in vitro. A, Effects of miR-145 on T24 cell migration as examined by wound healing assay (magnification, $\times 50$ ). B and $\mathrm{C}$, transwell assays used to evaluate the effects of miR-145 on T24 cell invasion B and migration C. Photographs of cell invasion and migration were acquired from polycarbonate membranes stained with crystal violet (magnification, ×200). Data were compared using independent samples t-test. 

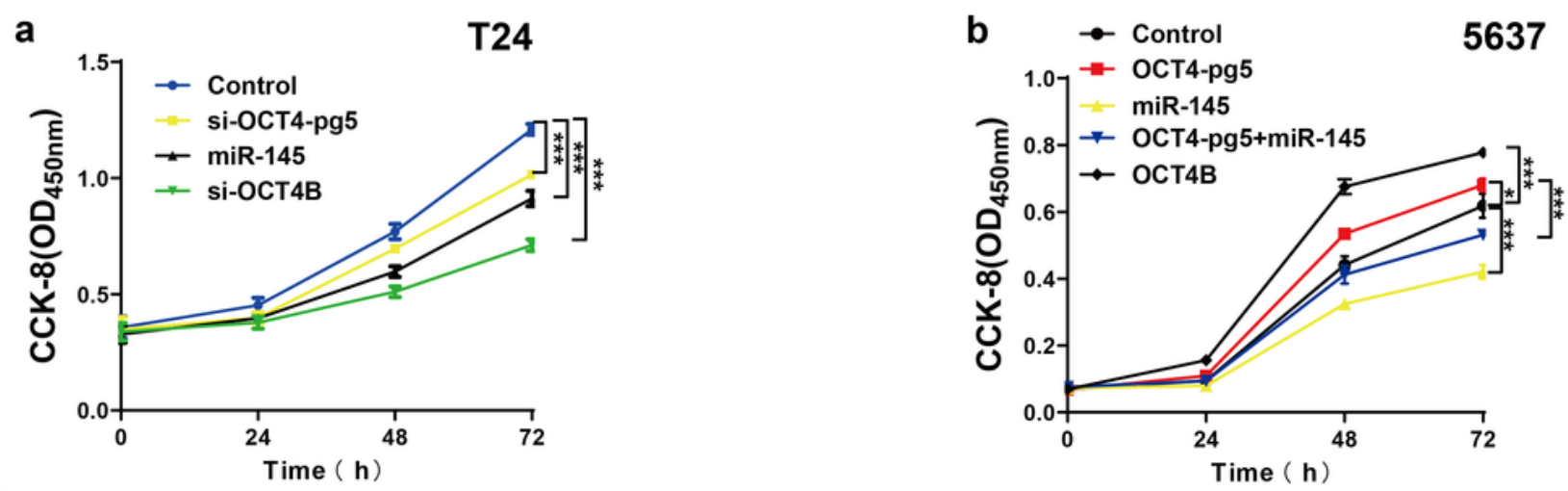

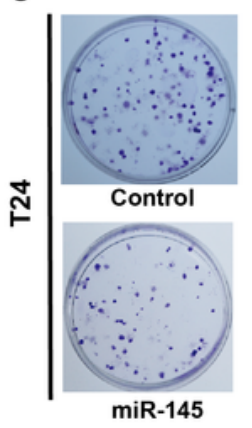

d

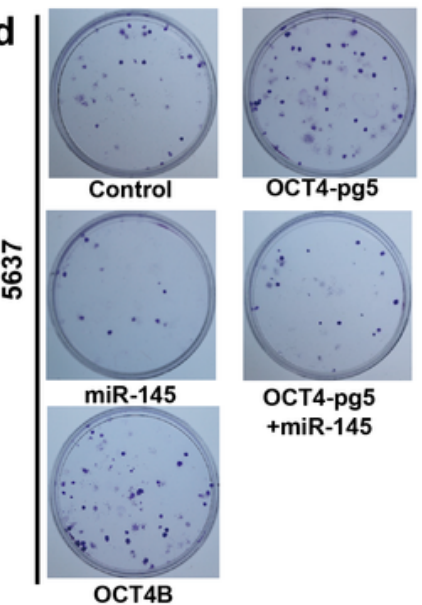

g

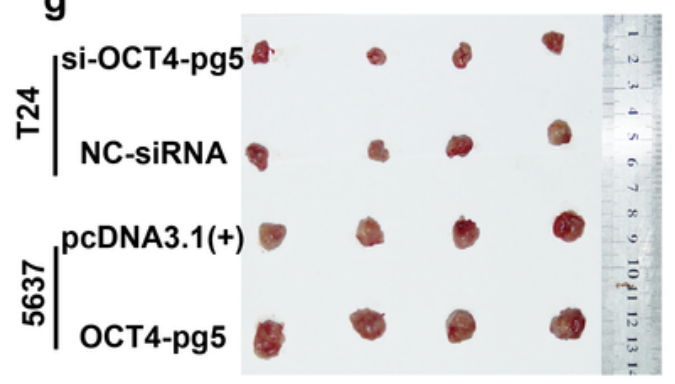

T24
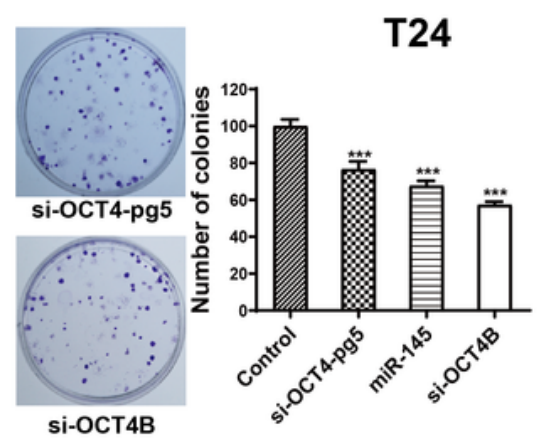

5637 e
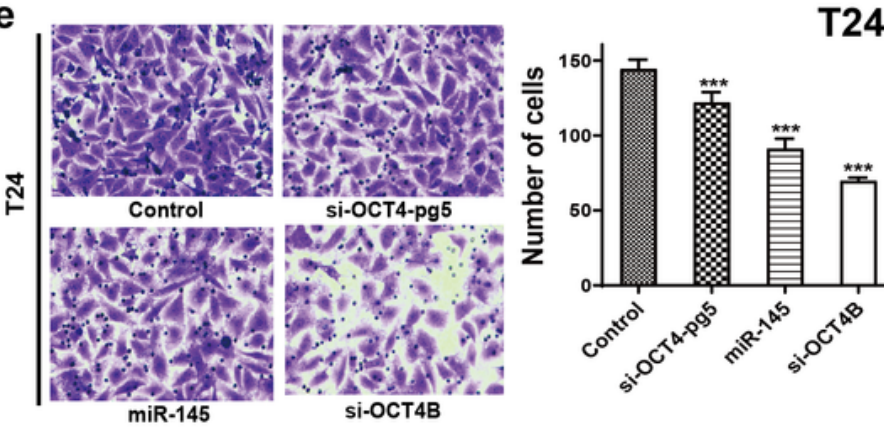

T24
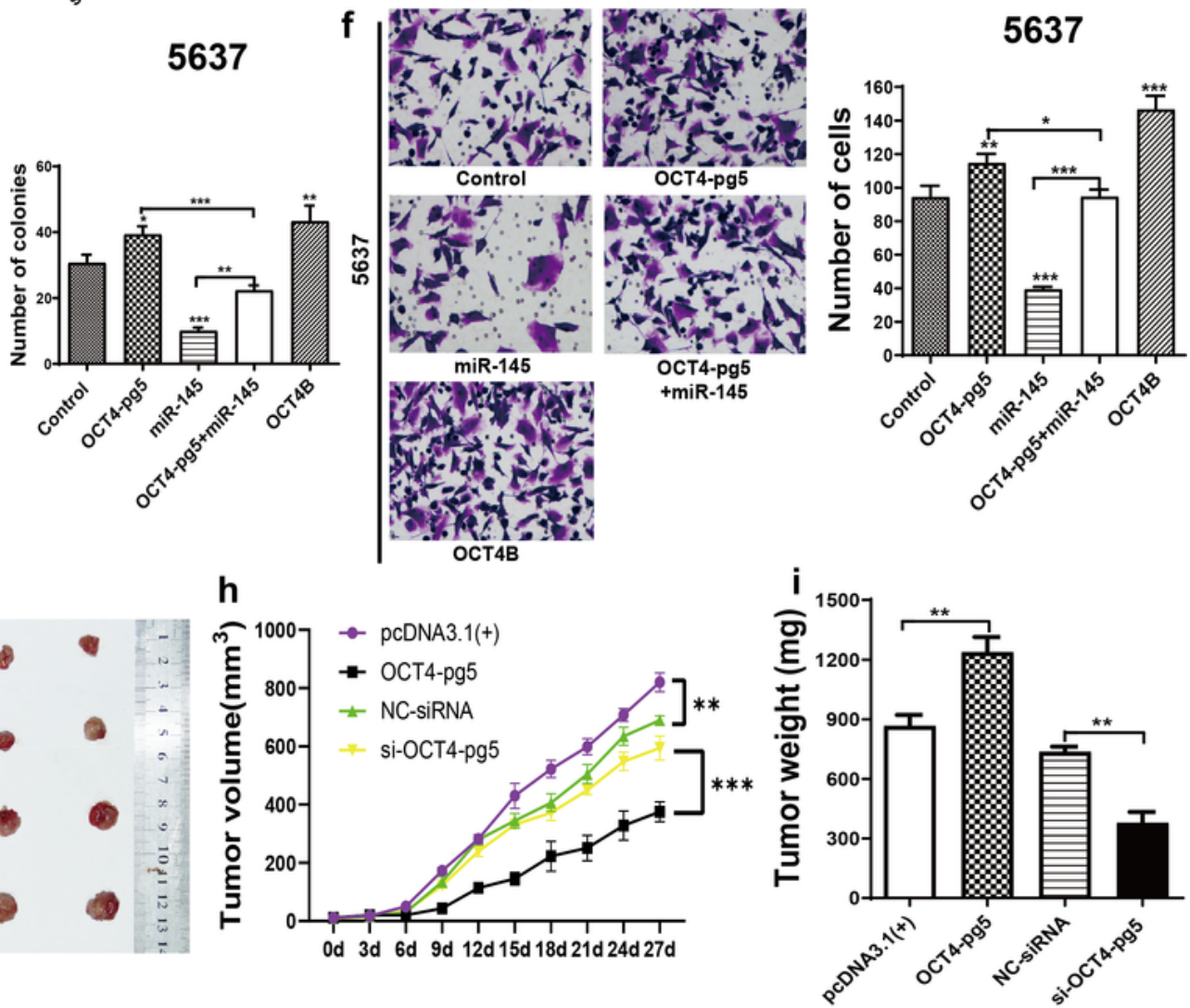

Figure 3

OCT4-pg5 and OCT4B function as oncogenes in BC cells in vitro and in vivo. A and B, CCK-8 assays showing viable T24 and 5637 cell numbers at different times post-transfection with the indicated transcripts. C and D, Colony formation assays to estimate the proliferation rates of T24 and $5637 \mathrm{BC}$ cells. E and F, Transwell assays to determine the invasion capacities of T24 and 5637 cells. G, Effect of OCT4-pg5 overexpression on tumor growth in vivo. H, Tumor growth curves measured every three days 
after inoculation of T24 or 5637 cells. I, Tumor weight was measured on the 27th day post-inoculation. Data were compared using independent samples t-test.

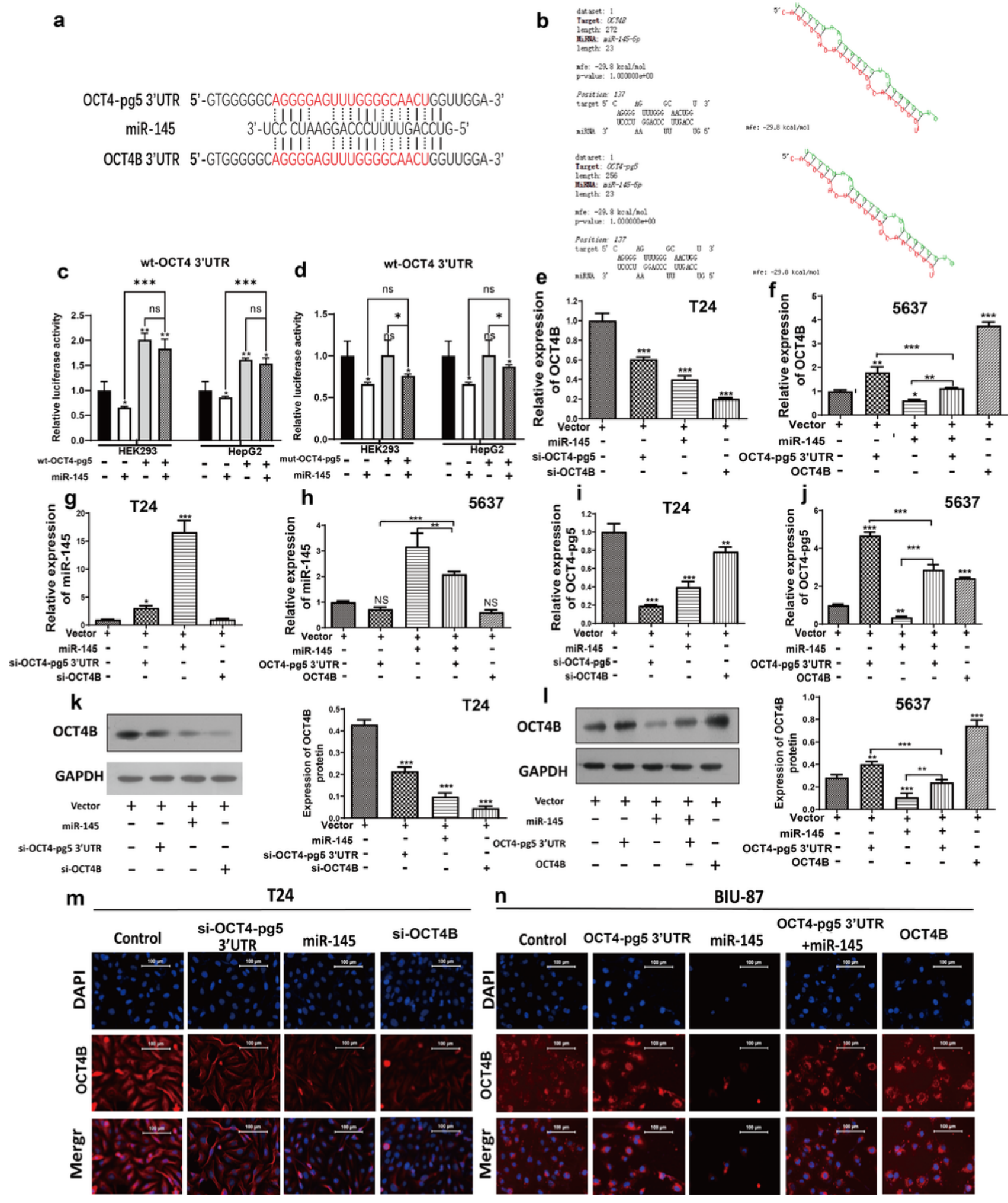

Figure 4

OCT4-pg5 3'UTR regulates the expression of OCT4B by sequestering miR-145. A, The sequence alignment of miR-145 with OCT4B and OCT4-pg5 3'UTRs. B, The binding force and possible secondary structure analyzed by RNAhybrid. C and D, Luciferase activity of OCT4B reporters in T24 and 5637 BC cells. MiR- 
145 mimics, wild-type, or mutant OCT4-pg5 3'UTR were co-transfected with luciferase reporters. E-F, Relative expression levels of OCT4B, miR-145, and OCT4-pg5 in T24 and 5637 cells were measured by qRT-PCR. $\mathrm{K}$ and L, Relative expression levels of OCT4B protein in T24 cells and 5637 cells as measured by Western blotting after the indicated transfection. Densitometric measures are shown to the right of each representative blot. M and N, Immunofluorescence analysis of OCT4B expression in T24 cells and 5637 cells transfected with the indicated vectors. Red: Anti-OCT4B. Blue: DAPI nuclear staining. Data were compared using independent samples t-test.

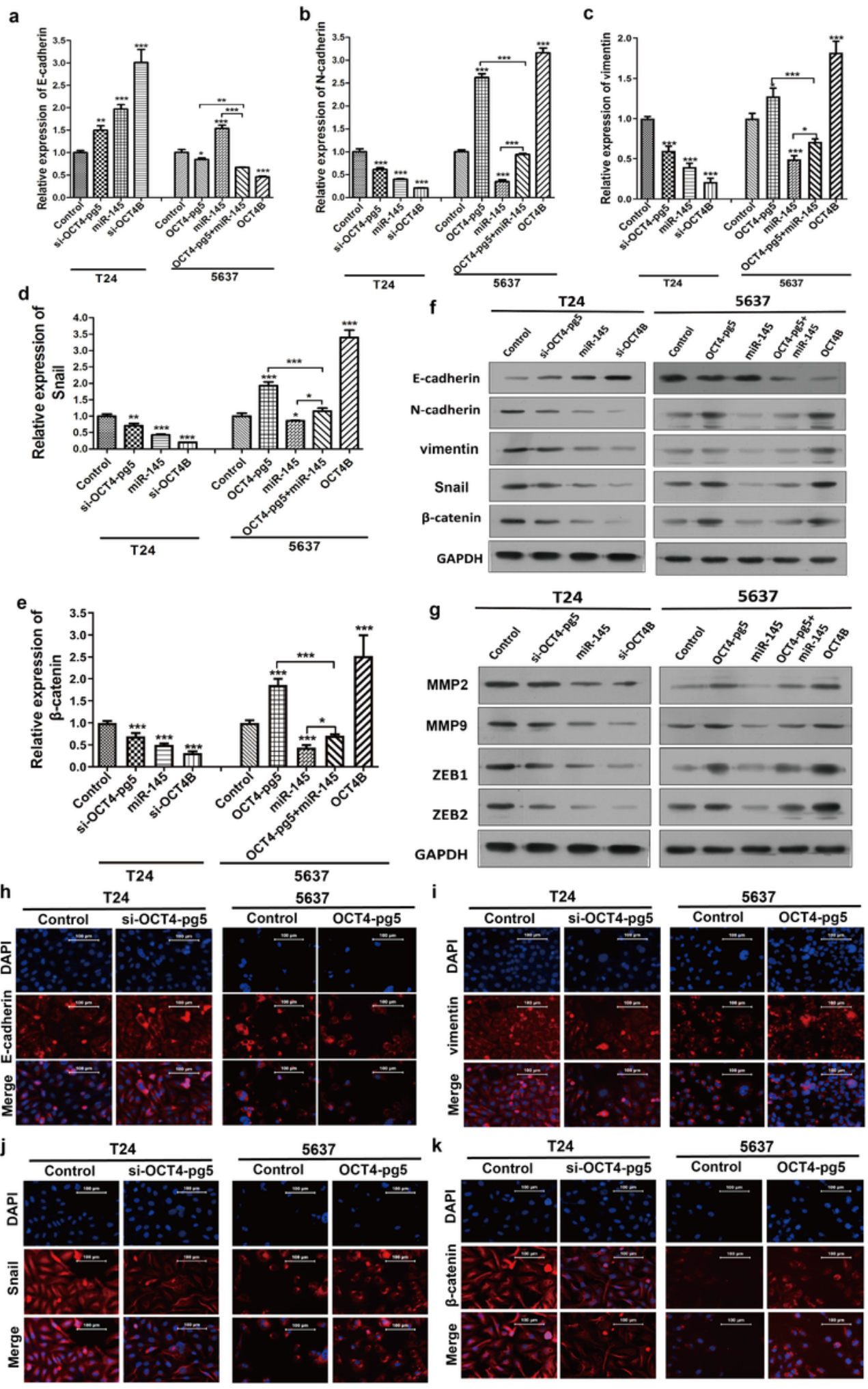




\section{Figure 5}

OCT4-pg5 promotes metastasis by inducing EMT through the Wnt/ $\beta$-catenin pathway. A-E, Relative mRNA expression levels of E-cadherin, vimentin, Snail and $\beta$-catenin in T24 cells and 5637 cells after transfection as measured by RT-PCR (normalized to $\beta$-actin). F and G, Relative protein expression levels of E-cadherin, vimentin, Snail, $\beta$-catenin, MMP2, MMP9, ZEB1 and ZEB2 in T24 cells and 5637 cells after transfection as measured by Western blot (normalized to GAPDH). H-K, Immunofluorescence staining for E-cadherin, vimentin, Snail and $\beta$-catenin in T24 cells and 5637 cells. Red: anti-E-cadherin, anti-vimentin, anti-Snail, and anti- $\beta$-catenin. Blue: DAPI nuclear staining. Data were compared using independent samples t-test.

\section{Image not available with this version}

\section{Figure 6}

Crosstalk between OCT4-pg5 and OCT4B enhances the resistance of T24 cells to cisplatin. A and B, OCT4-pg5 and OCT4B expression levels in T24 cells as measured by qRT-PCR, C, Western blot analysis of OCT4B protein level in T24 cells. GAPDH was used as the control. D, Effects of OCT4-pg5 3'UTR and OCT4B on the apoptotic rate of T24 cells as measured by flow cytometry. UL: necrotic cells, UR: terminal apoptotic cells, LR: early apoptotic cells, LL: normal cells. Cells in UR and LR were counted and analyzed. E, Effects of OCT4-pg5 and OCT4B expression on the cell cycle stage distribution of T24 cells as measured by flow cytometry. Data were compared using independent samples t-test.

\section{Supplementary Files}

This is a list of supplementary files associated with this preprint. Click to download.

- SupplementalTables.xls 